\title{
$K$-g-fusion frames in Hilbert spaces
}

Yongdong Huang ${ }^{1,2^{*}}$ and Yuanyuan Yang

${ }^{\text {*Correspondence: }}$

huang_yongdong@163.com

'School of Mathematics and Information Science, North Minzu

University, Yinchuan, China

${ }^{2}$ Center of Mathematics and

Information Science, Dalian Minzu

University, Dalian, China

\section{Springer}

\begin{abstract}
$K$-g-fusion frames are generalizations of fusion frames, $K$-fusion frames and $g$-fusion frames. In this paper, we present some new characterizations of $K$ - $g$-fusion frames and tight $\mathrm{K}$-g-fusion frames, and give several properties of $\mathrm{K}$ - $\mathrm{g}$-fusion frames by means of the methods and techniques in frame and operator theories. Then, we discuss the redundancy of $K$-g-fusion frames. Finally, we discuss the stability of $K$-g-fusion frames.
\end{abstract}

MSC: $42 \mathrm{C} 15$

Keywords: K-g-fusion frames; Bounded linear operator; Redundancy; Stability; Hilbert spaces

\section{Introduction}

Frames were first introduced by Duffin and Schaeffer [10] to study nonharmonic Fourier series in Hilbert spaces in 1952. In recent three decades, frames have been widely applied in various of fields of sampling theory, operator theory, signal processing, system modeling, data analysis, and so on; see $[3,7,8,12,20]$ for details. For special applications, various generalizations of frames were proposed, such as $K$-frames by Găvruta to study the atomic systems with respect to a bounded linear operator [11], g-frames by Sun as natural generalizations of frames [20], $K$-g-frames by Zhu [21] and Huang [13, 15] to fully combine the advantages of $K$-frame and g-frame in developing frame theory, fusion frames by Casazza and Kutyniok to model sensor network perfectly [4, 6], $K$-fusion frames by the authors of $[1,2]$ to generalize the theory of $K$-frames and fusion frames. $K$-g-fusion frames were proposed by Sadri and Rahimi [18], who discussed the duality and stability of $K$-g-fusion frames. In this paper, we present several properties and characterizations of $K$-g-fusion frames, and discuss the redundancy and stability of $K$-g-fusion frames.

Throughout this paper, $\mathcal{H}$ is a separable Hilbert space, $\left\{W_{j}\right\}_{j \in J}$ is a sequence of closed subspaces of $\mathcal{H}$, where $J$ is a subset of integers $\mathbb{Z} ; L\left(\mathcal{H}, W_{j}\right)$ is the collection of all bounded linear operators of $\mathcal{H}$ into $W_{j}$, with $L(\mathcal{H})=L(\mathcal{H}, \mathcal{H}) ; \pi_{W_{j}}$ denotes the orthogonal projection from $\mathcal{H}$ onto $W_{j}$. If $v_{j}>0$ for any $j \in J$, then we call $\left\{v_{j}\right\}_{j \in J}$ a family of weights. For $K \in L(\mathcal{H})$, $R(K)$ and $N(K)$ represent the range and null space of $K$, respectively; $I_{\mathcal{H}}$ is the identity operator on $\mathcal{H}$. The space $l^{2}\left(\left\{W_{j}\right\}_{j \in J}\right)$ is defined by

$$
l^{2}\left(\left\{W_{j}\right\}_{j \in J}\right)=\left\{\left\{f_{j}\right\}_{j \in J}: f_{j} \in W_{j}, \sum_{j \in J}\left\|f_{j}\right\|^{2}<\infty\right\}
$$

(c) The Author(s) 2020. This article is licensed under a Creative Commons Attribution 4.0 International License, which permits use, sharing, adaptation, distribution and reproduction in any medium or format, as long as you give appropriate credit to the original author(s) and the source, provide a link to the Creative Commons licence, and indicate if changes were made. The images or other third party material in this article are included in the article's Creative Commons licence, unless indicated otherwise in a credit line to the material. If material is not included in the article's Creative Commons licence and your intended use is not permitted by statutory regulation or exceeds the permitted use, you will need to obtain permission directly from the copyright holder. To view a copy of this licence, visit http://creativecommons.org/licenses/by/4.0/. 
with the inner product given by

$$
\left\langle\left\{f_{j}\right\},\left\{g_{j}\right\}\right\rangle=\sum_{j \in J}\left\langle f_{j}, g_{j}\right\rangle_{W_{j}}
$$

Then $l^{2}\left(\left\{W_{j}\right\}_{j \in J}\right)$ is Hilbert space with the pointwise operations.

\section{Preliminaries}

In this section, we mention some definitions and lemmas of generalizations of frames.

Definition 2.1 (Fusion frame [6]) Let $\left\{W_{j}\right\}_{j \in J}$ be a collection of closed subspaces in a Hilbert space $\mathcal{H}$, and let $\left\{v_{j}\right\}_{j \in J}$ be a family of weights. The family $\left\{\left(W_{j}, v_{j}\right)\right\}_{j \in J}$ is called a fusion frame for $\mathcal{H}$ if there exist constants $0<A \leq B<\infty$ such that

$$
A\|f\|^{2} \leq \sum_{j \in J} v_{j}^{2}\left\|\pi_{W_{j} f}\right\|^{2} \leq B\|f\|^{2}, \quad \forall f \in \mathcal{H}
$$

Definition 2.2 (g-fusion frame [5]) Let $\left\{W_{j}\right\}_{j \in J}$ be a collection of closed subspaces in a Hilbert space $\mathcal{H}$, and let $\left\{v_{j}\right\}_{j \in J}$ be a family of weights, $\Lambda_{j} \in L\left(\mathcal{H}, W_{j}\right)$ for any $j \in J$. The family $\left\{\left(W_{j}, \Lambda_{j}, v_{j}\right)\right\}_{j \in J}$ is called a g-fusion frame for $\mathcal{H}$ if there exist constants $0<A \leq B<\infty$ such that

$$
A\|f\|^{2} \leq \sum_{j \in J} v_{j}^{2}\left\|\Lambda_{j} \pi_{W_{j} f}\right\|^{2} \leq B\|f\|^{2}, \quad \forall f \in \mathcal{H}
$$

Definition 2.3 (K-g-fusion frame [18]) Let $K \in L(\mathcal{H})$ and $\left\{W_{j}\right\}_{j \in J}$ be a collection of closed subspaces in a Hilbert space $\mathcal{H}$, let $\left\{v_{j}\right\}_{j \in J}$ be a family of weights, $\Lambda_{j} \in L\left(\mathcal{H}, W_{j}\right)$ for any $j \in J$. The family $\left\{\left(W_{j}, \Lambda_{j}, v_{j}\right)\right\}_{j \in J}$ is called a $K$-g-fusion frame for $\mathcal{H}$ if there exist constants $0<A \leq B<\infty$ such that

$$
A\left\|K^{*} f\right\|^{2} \leq \sum_{j \in J} v_{j}^{2}\left\|\Lambda_{j} \pi_{W_{j}} f\right\|^{2} \leq B\|f\|^{2}, \quad \forall f \in \mathcal{H}
$$

We call $A, B$ lower and upper frame bounds of a $K$-g-fusion frame, respectively.

If the left-hand inequality of (2.3) is an equality, we say that $\left\{\left(W_{j}, \Lambda_{j}, v_{j}\right)\right\}_{j \in J}$ is a tight $K$-gfusion frame; if $A=1$, we say $\left\{\left(W_{j}, \Lambda_{j}, v_{j}\right)\right\}_{j \in J}$ is a Parseval $K$-g-fusion frame; $\left\{\left(W_{j}, \Lambda_{j}, v_{j}\right)\right\}_{j \in J}$ is called an exact $K$-g-fusion frame if it ceases to be a $K$-g-fusion frame whenever any one of its elements is removed; if only the right-hand inequality of (2.3) holds, $\left\{\left(W_{j}, \Lambda_{j}, v_{j}\right)\right\}_{j \in J}$ is called a g-fusion Bessel sequence with a bound $B$ for $\mathcal{H}$.

Remark 2.4 Let $\left\{\left(W_{j}, \Lambda_{j}, v_{j}\right)\right\}_{j \in J}$ be a $K$-g-fusion frame for $\mathcal{H}$. If $K=I_{\mathcal{H}}$, then $\left\{\left(W_{j}, \Lambda_{j}, v_{j}\right)\right\}_{j \in J}$ is a g-fusion frame for $\mathcal{H}$; if $\Lambda_{j}=\pi_{W_{j}}$ for any $j \in J$, then $\left\{\left(W_{j}, \Lambda_{j}, v_{j}\right)\right\}_{j \in J}\left(\left\{\left(W_{j}, v_{j}\right)\right\}_{j \in J}\right)$ is a $K$ fusion frame for $\mathcal{H}$; if $K=I_{\mathcal{H}}$ and $\Lambda_{j}=\pi_{W_{j}}$ for any $j \in J$, then $\left\{\left(W_{j}, \Lambda_{j}, v_{j}\right)\right\}_{j \in J}\left(\left\{\left(W_{j}, v_{j}\right)\right\}_{j \in J}\right)$ is a fusion frame for $\mathcal{H}$. Therefore, a $K$-g-fusion frame is a generalization of a fusion frame, g-fusion frame, and $K$-fusion frame. 
Now, we suppose that $\Lambda=\left\{\left(W_{j}, \Lambda_{j}, v_{j}\right)\right\}_{j \in J}$ is a g-fusion Bessel sequence with a bound $B$ for $\mathcal{H}$, and let $T_{\Lambda}$ denote a synthesis operator of $\Lambda$, that is, $T_{\Lambda}: l^{2}\left(\left\{W_{j}\right\}_{j \in J}\right) \rightarrow \mathcal{H}$,

$$
T_{\Lambda}\left(\left\{f_{j}\right\}_{j \in J}\right)=\sum_{j \in J} v_{j} \pi_{W_{j}} \Lambda_{j}^{*} f_{j}, \quad \forall\left\{f_{j}\right\}_{j \in J} \in l^{2}\left(\left\{W_{j}\right\}_{j \in J}\right) .
$$

The adjoint operator (analysis operator) $T_{\Lambda}^{*}: \mathcal{H} \rightarrow l^{2}\left(\left\{H_{j}\right\}_{j \in J}\right)$ is given by

$$
T_{\Lambda}^{*}(f)=\left\{v_{j} \Lambda_{j} \pi_{W_{j}} f\right\}_{j \in J}, \quad \forall f \in \mathcal{H} .
$$

Therefore, we can define the frame operator $S_{\Lambda}$ for a g-fusion Bessel sequence $\Lambda, S_{\Lambda}$ : $\mathcal{H} \rightarrow \mathcal{H}$ as follows:

$$
S_{\Lambda} f=T_{\Lambda} T_{\Lambda}^{*} f=\sum_{j \in J} v_{j}^{2} \pi_{W_{j}} \Lambda_{j}^{*} \Lambda_{j} \pi_{W_{j}} f, \quad \forall f \in \mathcal{H} .
$$

Then, we have

$$
\left\langle S_{A} f, f\right\rangle=\sum_{j \in J} v_{j}^{2}\left\|\Lambda_{j} \pi_{W_{j}} f\right\|^{2} \leq\langle B f, f\rangle, \quad \forall f \in \mathcal{H}
$$

or, $S_{\Lambda} \leq B I_{\mathcal{H}}$. Furthermore, if $\Lambda$ is a $K$-g-fusion frame with bounds $A$ and $B$, then we have

$$
A K K^{*} \leq S_{\Lambda} \leq B I_{\mathcal{H}}
$$

Inequalities (2.7) and (2.8) imply that $S_{\Lambda}$ a bounded, self-adjoint and positive operator. But we should note that $S_{\Lambda}$ for $K$-g-fusion frames is not invertible, in general. On the other hand, from [19], we know that if $K$ has closed range, then

$$
\left.S_{\Lambda}\right|_{R(K)}=S_{\Lambda}, \quad \forall f \in R(K)
$$

is invertible. Thus, for any $f \in R(K)$, we have the reconstruction formula

$$
f=\sum_{j \in J} v_{j}^{2} \pi_{W_{j}} \Lambda_{j}^{*} \Lambda_{j} \pi_{W_{j}}\left(\left.S_{\Lambda}\right|_{R(K)}\right)^{-1} f=\sum_{j \in J} v_{j}^{2}\left(\left.S_{\Lambda}\right|_{R(K)}\right)^{-1} \pi_{W_{j}} \Lambda_{j}^{*} \Lambda_{j} \pi_{W_{j}} f .
$$

From inequalities (2.6)-(2.8), we have

Proposition 2.5 Let $K \in L(\mathcal{H})$ and $\Lambda=\left\{\left(W_{j}, \Lambda_{j}, v_{j}\right)\right\}_{j \in J}$ be a $g$-fusion Bessel sequence for $\mathcal{H}$. Then $\Lambda$ is a K-g-fusion frame for $\mathcal{H}$ if and only if there exists a constant $A>0$ such that $A K K^{*} \leq S_{\Lambda}$, where $S_{\Lambda}$ is the frame operator for $\Lambda$.

Lemma 2.6 ([9]) Let $\mathcal{H}_{1}, \mathcal{H}_{2}$ and $\mathcal{H}$ be Hilbert spaces, $S \in L\left(\mathcal{H}_{1}, \mathcal{H}\right)$ and $T \in L\left(\mathcal{H}_{2}, \mathcal{H}\right)$. Then the following statements are equivalent:

(1) $R(S) \subseteq R(T)$.

(2) $S S^{*} \leq \alpha T T^{*}$ for some $\alpha>0$.

(3) $S=T M$ for some $M \in L\left(\mathcal{H}_{1}, \mathcal{H}_{2}\right)$.

By Lemma 2.6, we have the following proposition: 
Proposition 2.7 Let $K \in L(\mathcal{H})$ and $\Lambda=\left\{\left(W_{j}, \Lambda_{j}, v_{j}\right)\right\}_{j \in J}$ be a $K$-g-fusion frame for $\mathcal{H}$. Suppose that $T \in L(\mathcal{H})$ and $R(T) \subset R(K)$, then $\Lambda$ is a $T$-g-fusion frame for $\mathcal{H}$.

Lemma 2.8 ([16]) Let $W \subseteq \mathcal{H}$ be a closed subspace and $T \in L(\mathcal{H})$. Then

$$
\pi_{W} T^{*}=\pi_{W} T^{*} \pi_{T W} .
$$

Furthermore, if $T$ is a unitary operator, then

$$
\pi_{T W} T=T \pi_{W}
$$

Definition 2.9 ([14]) Let $S, T \in L(\mathcal{H})$. Then $S$ is said to be the Drazin inverse of $T$ if we have the following:

(1) $S T S=S$;

(2) $S T=T S$;

(3) $T S T^{k}=T^{k}$, for some positive integers $k$.

Lemma 2.10 ([7]) Let $\mathcal{H}$ be a Hilbert space, and suppose that $T \in L(\mathcal{H})$ has a closed range. Then there exists an operator $T^{\dagger} \in L(\mathcal{H})$ for which

$$
N\left(T^{\dagger}\right)=R(T)^{\perp}, \quad R\left(T^{\dagger}\right)=N(T)^{\perp}, \quad T T^{\dagger} y=y, \quad y \in R(T) .
$$

We call the operator $T^{\dagger}$ the pseudo-inverse of T. This operator is uniquely determined by these properties.

In fact, if $T$ is invertible, then we have $T^{-1}=T^{\dagger}$.

\section{Main results}

In this section, we present some new characterizations and properties of $K$-g-fusion frames and tight $K$-g-fusion frames, discuss the direct sum, redundancy and stability of $K$-g-fusion frames.

\subsection{Characterizations and properties of $K$-g-fusion frames}

In this section, we will give some new characterizations and properties of $K$-g-fusion frames and tight $K$-g-fusion frames.

Theorem 3.1 Let $K \in L(\mathcal{H})$ and $\Lambda=\left\{\left(W_{j}, \Lambda_{j}, v_{j}\right)\right\}_{j \in J}$ be a $g$-fusion Bessel sequence for $\mathcal{H}$ with frame operator $S_{\Lambda}$. Then $\Lambda$ is a $K$-g-fusion frame for $\mathcal{H}$ with respect to $\left\{\mathcal{H}_{j}\right\}_{j \in J}$ if and only if $K=S_{\Lambda}^{\frac{1}{2}} M$, for some $M \in L(\mathcal{H})$.

Proof First, we prove the necessity. Suppose $\Lambda$ is a $K$-g-fusion frame for $\mathcal{H}$, then there exists constant $A>0$ such that $A K K^{*} \leq S_{\Lambda}$, and $S_{\Lambda}$ is a self-adjoint and positive operator. Thus, $S_{\Lambda}^{\frac{1}{2}}$ is a self-adjoint and positive operator, so we have

$$
K K^{*} \leq \frac{1}{A} S_{\Lambda}^{\frac{1}{2}}\left(S_{\Lambda}^{\frac{1}{2}}\right)^{*}
$$

By Lemma 2.6, there exists some $M \in L(\mathcal{H})$ such that $K=S_{\Lambda}^{\frac{1}{2}} M$. 
Next, we prove the sufficiency. Suppose that there exists an operator $M \in L(\mathcal{H})$ so that $K=S_{\Lambda}^{\frac{1}{2}} M$. From Lemma 2.6, we know that $A K K^{*} \leq S_{\Lambda}$ holds for constant $A>0$. From Proposition 2.5, $\Lambda$ is a $K$-g-fusion frame.

Theorem 3.2 Let $K \in L(\mathcal{H})$ and $\Lambda=\left\{\left(W_{j}, \Lambda_{j}, v_{j}\right)\right\}_{j \in J}$ be a g-fusion Bessel sequence for $\mathcal{H}$. Suppose that $T_{\Lambda}$ is the synthesis operator of $\Lambda$. Then the following statements hold:

(1) If $\Lambda$ is a tight $K$-g-fusion frame for $\mathcal{H}$, then $R(K)=R\left(T_{\Lambda}\right)$.

(2) $R(K)=R\left(T_{\Lambda}\right)$ if and only if there exist two constants $A$ and $B$ such that

$$
A\left\|K^{*} f\right\|^{2} \leq \sum_{j \in J} v_{j}^{2}\left\|\Lambda_{j} \pi_{W_{j}} f\right\|^{2} \leq B\left\|K^{*} f\right\|^{2}, \quad \forall f \in \mathcal{H} .
$$

Proof (1) Suppose that $\Lambda$ is a tight $K$-g-fusion frame for $\mathcal{H}$, then there exists a constant $A>0$ such that

$$
A\left\|K^{*} f\right\|^{2}=\sum_{j \in J} v_{j}^{2}\left\|\Lambda_{j} \pi_{W_{j}} f\right\|^{2}=\left\|T_{\Lambda}^{*} f\right\|^{2}, \quad \forall f \in \mathcal{H} .
$$

Therefore, we have $A K K^{*}=T_{\Lambda} T_{\Lambda}^{*}$. By Lemma 2.6, we obtain $R(K)=R\left(T_{\Lambda}\right)$.

(2) First, we prove the necessity. Let $R(K)=R\left(T_{\Lambda}\right)$, using Lemma 2.6, there exist constants $A, B>0$ such that

$$
A K K^{*} \leq T_{\Lambda} T_{\Lambda}^{*} \leq B K K^{*}
$$

which implies that

$$
A\left\|K^{*} f\right\|^{2} \leq\left\|T_{\Lambda}^{*} f\right\|^{2}=\sum_{j \in J} v_{j}^{2}\left\|\Lambda_{j} \pi_{W_{j}} f\right\|^{2} \leq B\left\|K^{*} f\right\|^{2}, \quad \forall f \in \mathcal{H} .
$$

Therefore,

$$
A\left\|K^{*} f\right\|^{2} \leq \sum_{j \in J} v_{j}^{2}\left\|\Lambda_{j} \pi_{W_{j} f}\right\|^{2} \leq B\left\|K^{*} f\right\|^{2}, \quad \forall f \in \mathcal{H} .
$$

Next, we prove the sufficiency. Since $T_{\Lambda}$ is the synthesis operator of $\Lambda$, we can obtain

$$
\left\|T_{\Lambda}^{*} f\right\|^{2}=\sum_{j \in J} v_{j}^{2}\left\|\Lambda_{j} \pi_{W_{j}} f\right\|^{2} .
$$

Suppose that there exist constants $A, B>0$ such that

$$
A\left\|K^{*} f\right\|^{2} \leq \sum_{j \in J} v_{j}^{2}\left\|\Lambda_{j} \pi_{W_{j}} f\right\|^{2} \leq B\left\|K^{*} f\right\|^{2}, \quad \forall f \in \mathcal{H} .
$$

Consequently,

$$
A K K^{*} \leq T_{\Lambda} T_{\Lambda}^{*} \leq B K K^{*},
$$

So we finish the proof of Theorem 3.2. 
Theorem 3.3 Let $K \in L(\mathcal{H})$. Then the following statements hold:

(1) Every $g$-fusion frame is a $K$-g-fusion frame.

(2) If $R(K)$ is closed, every $K$-g-fusion frame is a $g$-fusion frame for $R(K)$.

Proof (1) Let $\Lambda=\left\{\left(W_{j}, \Lambda_{j}, v_{j}\right)\right\}_{j \in J}$ be a g-fusion frame for $\mathcal{H}$ with frame bounds $A$ and $B$. Then, for any $f \in \mathcal{H}$,

$$
\frac{A}{\|K\|^{2}}\left\|K^{*} f\right\|^{2} \leq A\|f\|^{2} \leq \sum_{j \in J} v_{j}^{2}\left\|\Lambda_{j} \pi_{W_{j}} f\right\|^{2} \leq B\|f\|^{2} .
$$

Thus, $\Lambda$ is a $K$-g-fusion frame with bounds $\frac{A}{\|K\|^{2}}$ and $B$.

(2) Let $\Lambda=\left\{\left(W_{j}, \Lambda_{j}, v_{j}\right)\right\}_{j \in J}$ be a $K$-g-fusion frame for $\mathcal{H}$ with frame bounds $A$ and $B$. If $R(K)$ is closed, by Lemma 2.10, there exists an operator $K^{\dagger} \in L(\mathcal{H})$ such that

$$
\left(K^{\dagger}\right)^{*} K^{*} f=f, \quad \forall f \in R(K) .
$$

Thus, for any $f \in R(K)$, we obtain

$$
\frac{A}{\left\|K^{\dagger}\right\|^{2}}\|f\|^{2} \leq A\left\|K^{*} f\right\|^{2} \leq \sum_{j \in J} v_{j}^{2}\left\|\Lambda_{j} \pi_{W_{j}} f\right\|^{2} \leq B\|f\|^{2} .
$$

So, $\Lambda$ is a g-fusion frame for $R(K)$ with bounds $\frac{A}{\left\|K^{\dagger}\right\|^{2}}$ and $B$.

Theorem 3.4 Let $K_{i} \in L(\mathcal{H})$ and $\Lambda=\left\{\left(W_{j}, \Lambda_{j}, v_{j}\right)\right\}_{j \in J}$ be a $K_{i}$-g-fusion frame for $\mathcal{H}$ with bounds $A_{i}$ and $B, i=1,2, \ldots, n$. Then, the following statements hold:

(1) If $a_{i}(i=1,2, \ldots, n)$ are not all zero, then $\Lambda$ is a $\sum_{i=1}^{n} a_{i} K_{i}$-g-fusion frame with bounds $\frac{1}{\max _{1 \leq i \leq n}\left|a_{i}\right|^{2} n \sum_{i=1}^{n} \frac{1}{A_{i}}}$ and $B$.

(2) $\Lambda$ is a $\prod_{i=1}^{n} K_{i}$-g-fusion frame with bounds $\frac{A_{n}}{\prod_{l=1}^{n-1}\left\|K_{l}\right\|^{2}}$ and $B$.

(3) If $K_{l} K_{i}=K_{i} K_{l}$ for any $l, i=1,2, \ldots, n$, then $\Lambda$ is a $\prod_{i=1}^{n} K_{i}$-g-fusion frame with bounds $\max _{1 \leq i \leq n} \frac{A_{i}}{\prod_{l=1, l \neq i}^{n}\left\|K_{l}\right\|^{2}}$ and $B$.

Proof (1) Since $\Lambda$ is a $K_{i}$-g-fusion frame with bounds $A_{i}$ and $B$, then we have

$$
A_{i}\left\|K_{i}^{*} f\right\|^{2} \leq \sum_{j \in J} v_{j}^{2}\left\|\Lambda_{j} \pi_{W_{j}} f\right\|^{2} \leq B\|f\|^{2}, \quad \forall f \in \mathcal{H},
$$

that is, for any $f \in \mathcal{H}$,

$$
\left\|K_{i}^{*} f\right\|^{2} \leq \frac{1}{A_{i}} \sum_{j \in J} v_{j}^{2}\left\|\Lambda_{j} \pi_{W_{j}} f\right\|^{2}, \quad i=1,2, \ldots, n .
$$

So we have

$$
\begin{aligned}
\left\|\sum_{i=1}^{n}\left(a_{i} K_{i}\right)^{*} f\right\|^{2} & \leq \max _{1 \leq i \leq n}\left|a_{i}\right|^{2}\left\|\sum_{i=1}^{n} K_{i}^{*} f\right\|^{2} \\
& \leq \max _{1 \leq i \leq n}\left|a_{i}\right|^{2} n \sum_{i=1}^{n}\left\|K_{i}^{*} f\right\|^{2}
\end{aligned}
$$




$$
\leq \max _{1 \leq i \leq n}\left|a_{i}\right|^{2}\left(n \sum_{i=1}^{n} \frac{1}{A_{i}} \sum_{j \in J} v_{j}^{2}\left\|\Lambda_{j} \pi_{W_{j}} f\right\|^{2}\right)
$$

that is,

$$
\frac{1}{\max _{1 \leq i \leq n}\left|a_{i}\right|^{2} n \sum_{i=1}^{n} \frac{1}{A_{i}}}\left\|\sum_{i=1}^{n}\left(a_{i} K_{i}\right)^{*} f\right\|^{2} \leq \sum_{j \in I} v_{j}^{2}\left\|\Lambda_{j} \pi_{W_{j}} f\right\|^{2} \leq B\|f\|^{2},
$$

which yields that $\Lambda$ is a $\sum_{i=1}^{n} a_{i} K_{i}$-g-fusion frame with bounds $\frac{1}{\max _{1 \leq i \leq n}\left|a_{i}\right|^{2} n \sum_{i=1}^{n} \frac{1}{A_{i}}}$ and $B$.

(2) By inequality (3.9), we have

$$
\begin{aligned}
\left\|\left(\prod_{l=1}^{n} K_{l}\right)^{*} f\right\|^{2} & \leq \prod_{l=1}^{n-1}\left\|K_{l}^{*}\right\|^{2}\left\|K_{n}^{*} f\right\|^{2} \\
& \leq \prod_{l=1}^{n-1}\left\|K_{l}^{*}\right\|^{2} \frac{1}{A_{n}} \sum_{j \in J} v_{j}^{2}\left\|\Lambda_{j} \pi_{W_{j}} f\right\|^{2},
\end{aligned}
$$

that is, for any $f \in \mathcal{H}$,

$$
\frac{A_{n}}{\prod_{l=1}^{n-1}\left\|K_{l}\right\|^{2}}\left\|\left(\prod_{l=1}^{n} K_{l}\right)^{*} f\right\|^{2} \leq \sum_{i \in I} v_{i}^{2}\left\|\Lambda_{i} \pi_{W_{i}} f\right\|^{2} \leq B\|f\|^{2},
$$

which implies that $\Lambda$ is a $\prod_{i=1}^{n} K_{i}$-g-fusion frame with bounds $\frac{A_{n}}{\prod_{l=1}^{n-1}\left\|K_{l}\right\|^{2}}$ and $B$.

(3) By inequality (3.9) and since $K_{l} K_{i}=K_{i} K_{l}$, we have

$$
\begin{aligned}
\left\|\left(\prod_{l=1}^{n} K_{l}\right)^{*} f\right\|^{2} & \leq \prod_{l=1, l \neq i}^{n}\left\|K_{l}^{*}\right\|^{2}\left\|K_{i}^{*} f\right\|^{2} \\
& \leq \prod_{l=1, l \neq i}^{n}\left\|K_{l}^{*}\right\|^{2} \frac{1}{A_{i}} \sum_{j \in J} v_{j}^{2}\left\|\Lambda_{j} \pi_{W_{j}} f\right\|^{2},
\end{aligned}
$$

that is, for any $f \in \mathcal{H}$,

$$
\frac{A_{i}}{\prod_{l=1, l \neq i}^{n}\left\|K_{l}\right\|^{2}}\left\|\left(\prod_{l=1}^{n} K_{l}\right)^{*} f\right\|^{2} \leq \sum_{j \in J} v_{j}^{2}\left\|\Lambda_{j} \pi_{W_{j}} f\right\|^{2} \leq B\|f\|^{2} .
$$

Letting $M=\max _{1 \leq i \leq n} \frac{A_{i}}{\prod_{l=1, l \neq i}^{n}\left\|K_{l}\right\|^{2}}$, then inequality (3.12) implies that $\Lambda$ is a $\prod_{i=1}^{n} K_{i}$-gfusion frame with bounds $M$ and $B$.

Theorem 3.5 Let $U, K \in L(\mathcal{H})$ and $U$ be surjective. Suppose that $\Lambda=\left\{\left(W_{j}, \Lambda_{j}, v_{j}\right)\right\}_{j \in J}$ is a $K$-g-fusion frame for $\mathcal{H}$ with frame bounds $A$ and $B$. Then the following statements hold:

(1) If $U K=K U$, then $\Phi=\left\{\left(U W_{j}, \Lambda_{j} \pi_{W_{j}} U^{*}, v_{j}\right)\right\}_{j \in J}$ is a $K$-g-fusion frame with bounds $A\left\|U^{\dagger}\right\|^{-2}$ and $B\|U\|^{2}$.

(2) If $U^{*} \Lambda_{j} \pi_{W_{j}}=\Lambda_{j} \pi_{W_{j}} U^{*}$, then $\Phi=\left\{\left(U W_{j}, \Lambda_{j} \pi_{W_{j}} U^{*}, v_{j}\right)\right\}_{j \in J}$ is a K-g-fusion frame with bounds $A\left\|U^{\dagger}\right\|^{-2}$ and $B\|U\|^{2}$. 
(3) If $U K=K U$ and $U^{*} \pi_{W_{j}}=\pi_{W_{j}} U^{*}$, then $\Phi_{0}=\left\{\left(W_{j}, \Lambda_{j} U^{*}, v_{j}\right)\right\}_{j \in J}$ is a $K$-g-fusion frame with bounds $A\left\|U^{\dagger}\right\|^{-2}$ and $B\|U\|^{2}$.

Proof Since $U$ is surjective, from Lemma 2.10, there exists an operator $U^{\dagger} \in L(H)$ such that $U U^{\dagger}=I_{\mathcal{H}}$, that is, $\left(U^{\dagger}\right)^{*} U^{*}=I_{\mathcal{H}}$. Therefore, for all $f \in \mathcal{H}$,

$$
\frac{\|f\|}{\left\|U^{\dagger}\right\|} \leq\left\|U^{*} f\right\| .
$$

(1) By Lemma 2.8, for all $f \in \mathcal{H}$, we have

$$
\begin{aligned}
\sum_{j \in J} v_{j}^{2}\left\|\Lambda_{j} \pi_{W_{j}} U^{*} \pi_{U W_{j}} f\right\|^{2} & =\sum_{j \in J} v_{j}^{2}\left\|\Lambda_{j} \pi_{W_{j}} U^{*} f\right\|^{2} \\
& \leq B\left\|U^{*} f\right\|^{2} \\
& \leq B\|U\|^{2}\|f\|^{2}
\end{aligned}
$$

Thus, $\Phi$ is a g-fusion Bessel sequence for $\mathcal{H}$. On the other hand, notice that $\Lambda$ is a $K$-gfusion frame and $U K=K U$, so by inequality (3.13), we have

$$
\begin{aligned}
\sum_{j \in J} v_{j}^{2}\left\|\Lambda_{j} \pi_{W_{j}} U^{*} \pi_{U W_{j}} f\right\|^{2} & =\sum_{j \in J} v_{j}^{2}\left\|\Lambda_{j} \pi_{W_{j}} U^{*} f\right\|^{2} \\
& \geq A\left\|K^{*} U^{*} f\right\|^{2}=A\left\|U^{*} K^{*} f\right\|^{2} \\
& \geq A\left\|U^{\dagger}\right\|^{-2}\left\|K^{*} f\right\|^{2} .
\end{aligned}
$$

Hence, $\Phi$ is a $K$-g-fusion frame with bounds $A\left\|U^{\dagger}\right\|^{-2}$ and $B\|U\|^{2}$.

(2) From part (1), we know that $\Phi$ is a g-fusion Bessel sequence for $\mathcal{H}$. On the other hand, for all $f \in \mathcal{H}$, notice that $U^{*} \Lambda_{j} \pi_{W_{j}}=\Lambda_{j} \pi_{W_{j}} U^{*}$, so by inequality (3.13), we have

$$
\begin{aligned}
\sum_{j \in J} v_{j}^{2}\left\|\Lambda_{j} \pi_{W_{j}} U^{*} \pi_{U W_{j}} f\right\|^{2} & =\sum_{j \in J} v_{j}^{2}\left\|\Lambda_{j} \pi_{W_{j}} U^{*} f\right\|^{2} \\
& =\sum_{j \in J} v_{j}^{2}\left\|U^{*} \Lambda_{j} \pi_{W_{j}} f\right\|^{2} \\
& \geq\left\|U^{\dagger}\right\|^{-2} \sum_{j \in J} v_{j}^{2}\left\|\Lambda_{j} \pi_{W_{j}} f\right\|^{2} \\
& \geq A\left\|U^{\dagger}\right\|^{-2}\left\|K^{*} f\right\|^{2} .
\end{aligned}
$$

Hence, $\Phi$ is a $K$-g-fusion frame for $\mathcal{H}$ with bounds $A\left\|U^{\dagger}\right\|^{-2}$ and $B\|U\|^{2}$.

(3) Noticing that $U^{*} \pi_{W_{j}}=\pi_{W_{j}} U^{*}$, for all $f \in \mathcal{H}$, we have

$$
\begin{aligned}
\sum_{j \in J} v_{j}^{2}\left\|\Lambda_{j} U^{*} \pi_{W_{j}} f\right\|^{2} & =\sum_{j \in J} v_{j}^{2}\left\|\Lambda_{j} \pi_{W_{j}} U^{*} f\right\|^{2} \\
& \leq B\left\|U^{*} f\right\|^{2} \\
& \leq B\|U\|^{2}\|f\|^{2} .
\end{aligned}
$$


On the other hand, by inequality (3.13), we obtain

$$
\begin{aligned}
\sum_{j \in J} v_{j}^{2}\left\|\Lambda_{j} U^{*} \pi_{W_{j}} f\right\|^{2} & =\sum_{j \in J} v_{j}^{2}\left\|\Lambda_{j} \pi_{W_{j}} U^{*} f\right\|^{2} \\
& \geq A\left\|K^{*} U^{*} f\right\|^{2} \\
& \geq A\left\|U^{\dagger}\right\|^{-2}\left\|K^{*} f\right\|^{2} .
\end{aligned}
$$

Hence, $\Phi_{0}$ is a $K$-g-fusion frame with bounds $A\left\|U^{\dagger}\right\|^{-2}$ and $B\|U\|^{2}$.

In terms of the isometry and coisometry operators, we can obtain the following properties of a $K$-g-fusion frame.

Corollary 3.6 Let $U, K \in L(\mathcal{H})$ and $U$ be a coisometry operator. Suppose that $\Lambda=$ $\left\{\left(W_{j}, \Lambda_{j}, v_{j}\right)\right\}_{j \in J}$ is a $K$-g-fusion frame for $\mathcal{H}$ with frame bounds $A$ and $B$. Then the following statements hold:

(1) If $U K=K U$, then $\Phi=\left\{\left(U W_{j}, \Lambda_{j} \pi_{W_{j}} U^{*}, v_{j}\right)\right\}_{j \in J}$ is a $K$-g-fusion frame with bounds $A$ and $B$.

(2) If $U^{*} \Lambda_{j} \pi_{W_{j}}=\Lambda_{j} \pi_{W_{j}} U^{*}$, then $\Phi=\left\{\left(U W_{j}, \Lambda_{j} \pi_{W_{j}} U^{*}, v_{j}\right)\right\}_{j \in J}$ is a $K$-g-fusion frame with bounds $A$ and $B$.

(3) If $U K=K U$ and $U^{*} \pi_{W_{j}}=\pi_{W_{j}} U^{*}$, then $\Phi_{0}=\left\{\left(W_{j}, \Lambda_{j} U^{*}, v_{j}\right)\right\}_{j \in J}$ is a $K$-g-fusion frame with bounds $A$ and $B$.

Proof If $U$ is a coisometry operator, then $U$ is surjective and $\|U\|=\left\|U^{\dagger}\right\|=1$, so the results of Corollary 3.6 are valid due to Theorem 3.5.

Theorem 3.7 Let $K, U \in L(\mathcal{H})$ and $\Lambda=\left\{\left(W_{j}, \Lambda_{j}, v_{j}\right)\right\}_{j \in J}$ be a $K$-g-fusion frame for $\mathcal{H}$ with bounds $A$ and $B$. Suppose that $U$ is an isometry. Then the following statements hold:

(1) If $T=K U^{*}$, then $\Lambda$ is a Parseval $K$-g-fusion frame for $\mathcal{H}$, where $T$ is the synthesis operator of $\Lambda$.

(2) $\Phi=\left\{\left(W_{j}, U \Lambda_{j}, v_{j}\right)\right\}_{j \in J}$ is also a $K$-g-fusion frame with bounds $A$ and $B$.

Proof (1) For any $f \in \mathcal{H}$, we have

$$
\sum_{j \in J} v_{j}^{2}\left\|\Lambda_{j} \pi_{W_{j}} f\right\|^{2}=\left\|T^{*} f\right\|^{2}=\left\|\left(K U^{*}\right)^{*} f\right\|^{2}=\left\|K^{*} f\right\|^{2}
$$

Thus, $\Lambda$ is a Parseval $K$-g-fusion frame.

(2) Since $\Lambda$ is a $K$-g-fusion frame, then, for any $f \in \mathcal{H}$, we have

$$
A\left\|K^{*} f\right\|^{2} \leq \sum_{j \in J} v_{j}^{2}\left\|U \Lambda_{j} \pi_{W_{j}} f\right\|^{2}=\sum_{j \in J} v_{j}^{2}\left\|\Lambda_{j} \pi_{W_{j}} f\right\|^{2} \leq B\|f\|^{2}
$$

It is obvious that $\Phi$ is a $K$-g-fusion frame for $\mathcal{H}$ with the same bounds as for $\Lambda$.

Corollary 3.8 Let $K, U \in L(\mathcal{H})$ and $\Lambda=\left\{\left(W_{j}, \Lambda_{j}, v_{j}\right)\right\}_{j \in J}$ be a $K$-g-fusion frame for $\mathcal{H}$ with bounds $A$ and $B$. Suppose that $U$ is a coisometry. Then the following statements hold: 
(1) If $T=K U$, then $\Lambda$ is a Parseval $K$-g-fusion frame for $\mathcal{H}$, where $T$ is the synthesis operator of $\Lambda$.

(2) $\Phi=\left\{\left(W_{j}, U^{*} \Lambda_{j}, v_{j}\right)\right\}_{j \in J}$ is also a $K-g$-fusion frame with bounds $A$ and $B$.

Like $K$-frame and $K$-g-frame, we can discuss the property of $K$-g-fusion frames by employing the nonzero Drazin inverse of operator $K$.

Theorem 3.9 Let $K \in L(\mathcal{H})$ and $\Lambda=\left\{\left(W_{j}, \Lambda_{j}, v_{j}\right)\right\}_{j \in J}$ be a $K$-g-fusion frame for $\mathcal{H}$ with bounds $A$ and $B$ for all $f \in \mathcal{H}$. If $S \in L(\mathcal{H})$ is a nonzero Drazin inverse of $K$, then $\Lambda=$ $\left\{\left(W_{j}, \Lambda_{j}, v_{j}\right)\right\}_{j \in J}$ is an $S K S^{r}$-g-fusion frame for $\mathcal{H}$ with bounds $\frac{A}{\|S\|^{2 r+2}}$ and $B$ for any positive integer $r$.

Proof Since $\Lambda$ is a $K$-g-fusion frame, then, for any $f \in \mathcal{H}$, we have

$$
A\left\|K^{*} f\right\|^{2} \leq \sum_{j \in J} v_{j}^{2}\left\|\Lambda_{j} \pi_{W_{j}} f\right\|^{2} \leq B\|f\|^{2} .
$$

Using Definition 2.9, we have

$$
\left\|\left(S K S^{r}\right)^{*} f\right\|^{2}=\left\|\left(S^{r+1}\right)^{*} K^{*} f\right\|^{2} \leq\|S\|^{2 r+2}\left\|K^{*} f\right\|^{2},
$$

which implies that, for any $f \in \mathcal{H}$,

$$
\begin{aligned}
\frac{A}{\|S\|^{2 r+2}}\left\|\left(S K S^{r}\right)^{*} f\right\|^{2} & \leq A\left\|K^{*} f\right\|^{2} \\
& \leq \sum_{j \in J} v_{j}^{2}\left\|\Lambda_{j} \pi_{W_{j}} f\right\|^{2} \\
& \leq B\|f\|^{2} .
\end{aligned}
$$

Thus, $\Lambda$ is an $S K S^{r}$-g-fusion frame for $\mathcal{H}$.

Corollary 3.10 Let $K \in L(\mathcal{H})$ and $\Lambda=\left\{\left(W_{j}, \Lambda_{j}, v_{j}\right)\right\}_{j \in J}$ be a $K$-g-fusion frame for $\mathcal{H}$ with bounds $A$ and $B$. If $S \in L(\mathcal{H})$ is an idempotent operator of the nonzero Drazin inverse of $K$, then $\Lambda$ is an $S K S^{r}$-g-fusion frame for $\mathcal{H}$ with bounds $\frac{A}{\|S\|}$ and $B$ for any positive integer $r$.

Corollary 3.11 Let $K \in L(\mathcal{H})$ and $\Lambda=\left\{\left(W_{j}, \Lambda_{j}, v_{j}\right)\right\}_{j \in J}$ be a $K$-g-fusion frame for $\mathcal{H}$ with bounds $A$ and $B$. If $S \in L(\mathcal{H})$ is a nonzero Drazin inverse of $K$, then $\Lambda$ is also an $S^{r} K-g$ fusion frame (or $K S^{r}$-g-fusion frame) for $\mathcal{H}$ with bounds $\frac{A}{\|S\|^{2 r}}$ and $B$ for any positive integer $r$.

In [17], the authors presented several results on $K$-frames using techniques on quotient of bounded operators. We intend to generalize some results to $K$-g-fusion frames and tight $K$-g-fusion frames. Letting $W, V \in L(\mathcal{H})$ with $N(V) \subset N(W)$, from [17], the quotient operator $T=[W / V]$ is a linear operator on $\mathcal{H}$ from $R(V)$ to $R(W)$ defined by $V x \mapsto W x$. In this case $D(T)=R(V), R(T) \subset R(W)$ and $T V=W$, where $D(T)$ is the domain of the operator $T$. In the following theorem, we present a necessary and sufficient condition of a $K$-g-fusion frame by the quotient operator. 
Theorem 3.12 Let $K \in L(\mathcal{H})$ and $\Lambda=\left\{\left(W_{j}, \Lambda_{j}, v_{j}\right)\right\}_{j \in J}$ be a $g$-fusion Bessel sequence for $\mathcal{H}$ with frame operator $S$. Then $\Lambda=\left\{\left(W_{j}, \Lambda_{j}, v_{j}\right)\right\}_{j \in J}$ a $K$-g-fusion frame if and only if the quotient operator $\left[K^{*} / S^{\frac{1}{2}}\right]$ is bounded.

Proof First, we prove the necessity. Since $\Lambda$ is a $K$-g-fusion frame, there exists a constant $A>0$ such that

$$
A\left\|K^{*} f\right\|^{2} \leq \sum_{j \in J} v_{j}^{2}\left\|\Lambda_{j} \pi_{W_{j}} f\right\|^{2}=\langle S f, f\rangle=\left\|S^{\frac{1}{2}} f\right\|^{2}, \quad \forall f \in \mathcal{H} .
$$

Now let us define the operator $T: R\left(S^{\frac{1}{2}}\right) \rightarrow R\left(K^{*}\right)$ such that

$$
T\left(S^{\frac{1}{2}} f\right)=K^{*} f, \quad \forall f \in \mathcal{H},
$$

which implies that $N\left(S^{\frac{1}{2}}\right) \subset N\left(K^{*}\right)$ and $T$ is a linear operator. Using inequalities (3.17) and (3.18), we obtain

$$
\left\|T\left(S^{\frac{1}{2}} f\right)\right\|=\left\|K^{*} f\right\| \leq \frac{1}{\sqrt{A}}\left\|S^{\frac{1}{2}} f\right\| .
$$

Hence, $T$ is a bounded linear operator.

Now we prove sufficiency. Supposed that the quotient operator $\left[K^{*} / S^{\frac{1}{2}}\right]$ is bounded. Then there exists a constant $B>0$ such that

$$
\left\|K^{*} f\right\|^{2} \leq B\left\|S^{\frac{1}{2}} f\right\|^{2}=B\langle S f, f\rangle=B \sum_{j \in J} v_{j}^{2}\left\|\Lambda_{j} \pi_{W_{j}} f\right\|^{2}, \quad \forall f \in \mathcal{H} .
$$

Therefore, $\Lambda$ is a $K$-g-fusion frame for $\mathcal{H}$.

For tight $K$-g-fusion frames, we have the following result.

Theorem 3.13 Let $K \in L(\mathcal{H})$ and $\Lambda=\left\{\left(W_{j}, \Lambda_{j}, v_{j}\right)\right\}_{j \in J}$ be a tight $K$-g-fusion frame for $\mathcal{H}$ with the frame operator $S$. Then the quotient operator $\left[K^{*} / S^{\frac{1}{2}}\right]$ is invertible.

Proof Since $\Lambda$ is a tight $K$-g-fusion frame, by its definition and properties, there exists a constant $\alpha>0$ such that $S=\alpha K K^{*}$. Using Lemma 2.6, we can also get $R(K)=R\left(S^{\frac{1}{2}}\right)$. Denote $K_{0}=\left.K\right|_{N(K)^{\perp}}$, then $K_{0}^{-1}: R(K) \rightarrow N(K)^{\perp}$ is a closed linear operator. Let us define $Q:=K_{0}^{-1} S^{\frac{1}{2}}$ from $\mathcal{H}$ onto $N(K)^{\perp}$. Obviously, $S^{\frac{1}{2}}=K Q$ and $Q$ is bounded by the Closed Graph Theorem. Thus, we can obtain a bounded operator $P: \mathcal{H} \rightarrow N\left(S^{\frac{1}{2}}\right)^{\perp}$ such that $K=$ $S^{\frac{1}{2}} P$.

Letting $x \in N\left(S^{\frac{1}{2}}\right)^{\perp}$, we have

$$
S^{\frac{1}{2}} x=K Q x=S^{\frac{1}{2}} P Q x,
$$

or equivalently,

$$
P Q x=x, \quad \forall x \in N\left(S^{\frac{1}{2}}\right)^{\perp} .
$$


Hence $P Q$ is the identity operator on $N\left(S^{\frac{1}{2}}\right)^{\perp}$. Similarly, $Q P$ is the identity operator on $N(K)^{\perp}$. It follows that

$$
\left.P\right|_{N(K)^{\perp}}: N(K)^{\perp} \rightarrow N\left(S^{\frac{1}{2}}\right)^{\perp}
$$

is an invertible operator. Therefore, $K^{*}=\left(\left.P\right|_{N(K)^{\perp}}\right)^{*} S^{\frac{1}{2}}$, which means that $\left[K^{*} / S^{\frac{1}{2}}\right]$ is invertible.

\subsection{Direct sum of $K$-g-fusion frames}

Now, we suppose that $\left\{\mathcal{H}_{i}\right\}_{i=1}^{n}$ is a sequence of mutually orthogonal closed subspaces of $\mathcal{H}$. Then, the direct sum $\bigoplus_{i=1}^{n} \mathcal{H}_{i}=\mathcal{H}_{1} \oplus \mathcal{H}_{2} \oplus \cdots \oplus \mathcal{H}_{n}$ is also a Hilbert space. Let $T_{i} \in L\left(\mathcal{H}_{i}\right)$, $1 \leq i \leq n$ and consider the operator $\bigoplus_{i=1}^{n} T_{i}: \bigoplus_{i=1}^{n} \mathcal{H}_{i} \rightarrow \bigoplus_{i=1}^{n} \mathcal{H}_{i}$ defined by

$$
\left(\bigoplus_{i=1}^{n} T_{i}\right)\left(\bigoplus_{i=1}^{n} x_{i}\right)=T_{1} x_{1} \oplus T_{2} x_{2} \oplus \cdots \oplus T_{n} x_{n}=\sum_{i=1}^{n} T_{i} x_{i}, \quad \forall x_{i} \in \mathcal{H}_{i},
$$

where $\bigoplus_{i=1}^{n} T_{i}=T_{1} \oplus T_{2} \oplus \cdots \oplus T_{n}$. Then, $\bigoplus_{i=1}^{n} T_{i}$ is a bounded linear operator on $\bigoplus_{i=1}^{n} \mathcal{H}_{i}$. The conjugate operator on direct sums is defined by $\left(T_{1} \oplus T_{2}\right)^{*}=T_{1}^{*} \oplus T_{2}^{*}$.

In the following theorem, we will show a sufficient condition for a direct sum of $K$-gfusion frames be also a $K$-g-fusion frame.

Theorem 3.14 Let $\left\{\mathcal{H}_{j}\right\}_{j=1}^{n}$ be a sequence of mutually orthogonal closed subspaces of $\mathcal{H}$, and $\left\{\left(W_{i j}, \Lambda_{i j}, v_{i}\right)\right\}_{i \in I}$ be $K_{j}$-g-fusion frames for $\mathcal{H}_{j}, j=1,2, \ldots, n$. Then $\left\{\left(\bigoplus_{j=1}^{n} W_{i j}, \bigoplus_{j=1}^{n} \Lambda_{i j}, v_{i}\right)\right\}_{i \in I}$ is a $\bigoplus_{j=1}^{n} K_{j}$-g-fusion frame for $\bigoplus_{j=1}^{n} \mathcal{H}_{j}$.

Proof We only prove the result when $n=2$. Suppose that $\left\{\left(W_{i j}, \Lambda_{i j}, v_{i}\right)\right\}_{i \in I}$ is a $K_{j}$-g-fusion frame for $\mathcal{H}_{j}$ with bounds $A_{j}$ and $B_{j}, j=1,2$. Let $A=\min \left\{A_{1}, A_{2}\right\}, B=\max \left\{B_{1}, B_{2}\right\}$. Then for any $f \in \mathcal{H}_{1}$ and $g \in \mathcal{H}_{2}$, we have

$$
\begin{aligned}
A\left\|\left(K_{1} \oplus K_{2}\right)^{*}(f \oplus g)\right\|^{2}= & A\left\|\left(K_{1}^{*} \oplus K_{2}^{*}\right)(f \oplus g)\right\|^{2} \\
= & A\left\|K_{1}^{*} f \oplus K_{2}^{*} g\right\|^{2} \\
= & A\left(\left\|K_{1}^{*} f\right\|^{2}+\left\|K_{2}^{*} g\right\|^{2}\right) \\
\leq & A_{1}\left\|K_{1}^{*} f\right\|^{2}+A_{2}\left\|K_{2}^{*} g\right\|^{2} \\
\leq & \sum_{i \in I} v_{i}^{2}\left\|\Lambda_{i 1} \pi_{W_{i 1}}(f)\right\|^{2} \\
& +\sum_{i \in I} v_{i}^{2}\left\|\Lambda_{i 2} \pi_{W_{i 2}}(g)\right\|^{2} \\
\leq & B_{1}\|f\|^{2}+B_{2}\|g\|^{2} \\
\leq & B\left(\|f\|^{2}+\|g\|^{2}\right) \\
= & B\|f \oplus g\|^{2} .
\end{aligned}
$$


On the other hand, since $\mathcal{H}_{1}$ is orthogonal to $\mathcal{H}_{2}, \pi_{W_{i 1} \oplus W_{i 2}}=\pi_{W_{i 1}} \oplus \pi_{W_{i 2}}$ for any $i \in I$, so we have

$$
\sum_{i \in I} v_{i}^{2}\left\|\Lambda_{i 1} \pi_{W_{i 1}}(f)\right\|^{2}+\sum_{i \in I} v_{i}^{2}\left\|\Lambda_{i 2} \pi_{W_{i 2}}(g)\right\|^{2}=\sum_{i \in I} v_{i}^{2}\left\|\left(\Lambda_{i 1} \oplus \Lambda_{i 2}\right) \pi_{W_{i 1} \oplus W_{i 2}}(f \oplus g)\right\|^{2} .
$$

From the above two formulas, we know that $\left\{\left(\bigoplus_{j=1}^{n} W_{i j}, \bigoplus_{j=1}^{n} \Lambda_{i j}, v_{i}\right)\right\}_{i \in I}$ is a $\bigoplus_{j=1}^{n} K_{j}$-gfusion frame for $\bigoplus_{j=1}^{n} \mathcal{H}_{j}$.

\subsection{The redundancy of $\mathrm{K}$-g-fusion frames}

One of the important properties of frame theory is the possibility of redundancy. Many scholars discussed the redundancy of a frame, $\mathrm{g}$-frame, and fusion frame. In the following theorem, we discuss a sufficient condition that some elements can be removed without destroying the $K$-g-fusion frame property of the remaining set.

Theorem 3.15 Assume $K \in L(\mathcal{H})$ has closed range and $\left\{\left(W_{j}, \Lambda_{j}, v_{j}\right)\right\}_{j \in J}$ is a $K$-g-fusion frame for $\mathcal{H}$ with bounds $A$ and $B$. If $\overline{\operatorname{span}}\left\{W_{j}\right\}_{j \in J \backslash I} \subset R(K)$ and $\overline{\operatorname{span}}\left\{W_{j}\right\}_{j \in I} \perp R(K)$, where $I \subset J$, then $\left\{\left(W_{j}, \Lambda_{j}, v_{j}\right)\right\}_{j \in J \backslash I}$ is a $K$-g-fusion frame for $\mathcal{H}$ with bounds $A$ and $B$.

Proof Since $\overline{\operatorname{span}}\left\{W_{j}\right\}_{j \in I} \perp R(K)$, for any $g \in R(K) \subset \mathcal{H}$, we get $\pi_{W_{j}} g=0, j \in I$ and

$$
\sum_{j \in I} v_{j}\left\|\Lambda_{j} \pi_{W_{j}} g\right\|^{2}=0
$$

which implies that

$$
\begin{aligned}
\sum_{j \in J \backslash I} v_{j}\left\|\Lambda_{j} \pi_{W_{j}} g\right\|^{2} & =\sum_{j \in J} v_{j}\left\|\Lambda_{j} \pi_{W_{j}} g\right\|^{2}-\sum_{j \in I} v_{j}\left\|\Lambda_{j} \pi_{W_{j}} g\right\|^{2} \\
& =\sum_{j \in J} v_{j}\left\|\Lambda_{j} \pi_{W_{j}} g\right\|^{2} .
\end{aligned}
$$

Because $\left\{\left(W_{j}, \Lambda_{j}, v_{j}\right)\right\}_{j \in J}$ is a $K$-g-fusion frame for $\mathcal{H}$, we have

$$
A\left\|K^{*} f\right\|^{2} \leq \sum_{j \in J} v_{j}^{2}\left\|\Lambda_{j} \pi_{W_{j}} f\right\|^{2} \leq B\|f\|^{2}, \quad \forall f \in \mathcal{H} .
$$

Then, for any $g \in R(K)$, we have

$$
A\left\|K^{*} g\right\|^{2} \leq \sum_{j \in J \backslash I} v_{j}^{2}\left\|\Lambda_{j} \pi_{W_{j}} g\right\|^{2} .
$$

On the other hand, for any $g \in R(K)^{\perp}$ and for any $h \in \mathcal{H}$, we have

$$
\left\langle K^{*} g, h\right\rangle=\langle g, K h\rangle=0 .
$$

This yields

$$
K^{*} g=0, \quad g \in R(K)^{\perp} .
$$


Since $K$ has closed range, for any $g \in \mathcal{H}$, we have $g=g_{1}+g_{2}$, where $g_{1} \in R(K), g_{2} \in R(K)^{\perp}$. Due to $\overline{\operatorname{span}}\left\{W_{j}\right\}_{j \in J \backslash I} \subset R(K)$, we obtian $g_{2} \perp \overline{\operatorname{span}}\left\{W_{j}\right\}_{j \in J \backslash I}$ and

$$
\pi_{W_{j}} g_{2}=0, \quad j \in J \backslash I
$$

Then, for all $g \in \mathcal{H}$, using equations (3.25)-(3.27), we have

$$
A\left\|K^{*} g\right\|^{2}=A\left\|K^{*} g_{1}\right\|^{2} \leq \sum_{j \in J \backslash I} v_{j}\left\|\Lambda_{j} \pi_{W_{j}} g_{1}\right\|^{2}=\sum_{j \in J \backslash I} v_{j}\left\|\Lambda_{j} \pi_{W_{j}} g\right\|^{2} \leq B\|g\|^{2} .
$$

Therefore, $\left\{\left(W_{j}, \Lambda_{j}, v_{j}\right)\right\}_{j \in \backslash \backslash I}$ is a $K$-g-fusion frame for $\mathcal{H}$ with bounds $A$ and $B$.

Corollary 3.16 Let $K \in L(\mathcal{H})$ have closed range and $\left\{\left(W_{j}, \Lambda_{j}, v_{j}\right)\right\}_{j \in J}$ be a $K$-g-fusion frame for $\mathcal{H}$ with bounds $A$ and $B$. If $\overline{\operatorname{span}}\left\{W_{j}\right\}_{j \in J, j \neq j_{0}} \subset R(K)$ and $\overline{\operatorname{span}}\left\{W_{j_{0}}\right\} \perp R(K)$, where $j_{0} \in J$, then $\left\{\left(W_{j}, \Lambda_{j}, v_{j}\right)\right\}_{j \in J, j \neq j j_{0}}$ is a $K$-g-fusion frame for $\mathcal{H}$ with bounds $A$ and $B$.

\subsection{The stability of $K$-g-fusion frames}

Stability of a frame is an important research topic for frame theory. Many researchers discussed the stability of frames, fusion frames, $K$-frames, and $K$-g-frames $[3,7,8,13,14$, 16]. In the following theorems, we give some results about perturbations of $K$-g-fusion frame.

Theorem 3.17 Let $K \in L(\mathcal{H})$ and $\Lambda=\left\{\left(W_{j}, \Lambda_{j}, v_{j}\right)\right\}_{j \in J}$ be a $K$-g-fusion frame with bounds $A$ and B. Suppose $\Phi_{j} \in L\left(\mathcal{H}, W_{j}\right), j \in J$. If there exist constants $a, b, c$ satisfying $0 \leq a, b<$ $1,0 \leq \frac{c}{1-a}<\sqrt{A}$ and such that for any $f \in \mathcal{H}$,

$$
\begin{aligned}
\left(\sum_{j \in J} v_{j}^{2}\left\|\left(\Lambda_{j} \pi_{W_{j}}-\Phi_{j} \pi_{W_{j}}\right) f\right\|^{2}\right)^{\frac{1}{2}} \leq & a\left(\sum_{j \in J} v_{j}^{2}\left\|\Lambda_{j} \pi_{W_{j}} f\right\|^{2}\right)^{\frac{1}{2}} \\
& +b\left(\sum_{j \in J} v_{j}^{2}\left\|\Phi_{j} \pi_{W_{j}} f\right\|^{2}\right)^{\frac{1}{2}}+c\left\|K^{*} f\right\|,
\end{aligned}
$$

then $\Phi=\left\{\left(W_{j}, \Phi_{j}, v_{j}\right)\right\}_{j \in J}$ is also a $K$-g-fusion frame with bounds $\left(\frac{(1-a) \sqrt{A}-c}{1+b}\right)^{2}$ and $\left(\frac{(1+a) \sqrt{B}+c\|K\|}{1-b}\right)^{2}$ for $\mathcal{H}$.

Proof Since $\Lambda$ is a $K$-g-fusion frame with bounds $A$ and $B$ for $\mathcal{H}$, for any $f \in \mathcal{H}$, we have

$$
A\left\|K^{*} f\right\|^{2} \leq \sum_{j \in J} v_{j}^{2}\left\|\Lambda_{j} \pi_{W_{j}} f\right\|^{2} \leq B\|f\|^{2} .
$$

According to inequality (3.28), we have

$$
\begin{aligned}
\left(\sum_{j \in J} v_{j}^{2}\left\|\Phi_{j} \pi_{W_{j}} f\right\|^{2}\right)^{\frac{1}{2}} \leq & \left(\sum_{j \in J} v_{j}^{2}\left\|\left(\Phi_{j} \pi_{W_{j}}-\Lambda_{j} \pi_{W_{j}}\right) f\right\|^{2}\right)^{\frac{1}{2}} \\
& +\left(\sum_{j \in J} v_{j}^{2}\left\|\Lambda_{j} \pi_{W_{j}} f\right\|^{2}\right)^{\frac{1}{2}}
\end{aligned}
$$




$$
\begin{aligned}
\leq & (1+a)\left(\sum_{j \in J} v_{j}^{2}\left\|\Lambda_{j} \pi_{W_{j}} f\right\|^{2}\right)^{\frac{1}{2}} \\
& +b\left(\sum_{j \in J} v_{j}^{2}\left\|\Phi_{j} \pi_{W_{j}} f\right\|^{2}\right)^{\frac{1}{2}}+c\left\|K^{*} f\right\| .
\end{aligned}
$$

Hence,

$$
\begin{aligned}
\sum_{j \in J} v_{j}^{2}\left\|\Phi_{j} \pi_{W_{j}} f\right\|^{2} & \leq\left(\frac{1+a}{1-b}\left(\sum_{j \in J} v_{j}^{2}\left\|\Lambda_{j} \pi_{W_{j} f}\right\|^{2}\right)^{\frac{1}{2}}+\frac{c}{1-b}\left\|K^{*} f\right\|\right)^{2} \\
& \leq\left(\frac{(1+a) \sqrt{B}+c\|K\|}{1-b}\right)^{2}\|f\|^{2}, \quad \forall f \in \mathcal{H} .
\end{aligned}
$$

On the other hand, for any $f \in H$, we have

$$
\begin{aligned}
\left(\sum_{j \in J} v_{j}^{2}\left\|\Lambda_{j} \pi_{W_{j}} f\right\|^{2}\right)^{\frac{1}{2}} \leq & \left(\sum_{j \in J} v_{j}^{2}\left\|\left(\Lambda_{j} \pi_{W_{j}}-\Phi_{j} \pi_{W_{j}}\right) f\right\|^{2}\right)^{\frac{1}{2}} \\
& +\left(\sum_{j \in J} v_{j}^{2}\left\|\Phi_{j} \pi_{W_{j}} f\right\|^{2}\right)^{\frac{1}{2}} \\
\leq & a\left(\sum_{j \in J} v_{j}^{2}\left\|\Lambda_{j} \pi_{W_{j}} f\right\|^{2}\right)^{\frac{1}{2}} \\
& +(1+b)\left(\sum_{j \in J} v_{j}^{2}\left\|\Phi_{j} \pi_{W_{j}} f\right\|^{2}\right)^{\frac{1}{2}} \\
& +c\left\|K^{*} f\right\| .
\end{aligned}
$$

Consequently,

$$
\left(\frac{(1-a) \sqrt{A}-c}{1+b}\right)^{2}\left\|K^{*} f\right\|^{2} \leq \sum_{j \in J} v_{j}^{2}\left\|\Phi_{j} \pi_{W_{j}} f\right\|^{2}, \quad \forall f \in \mathcal{H}
$$

Using inequalities (3.29) and (3.30), we obtain that $\Phi=\left\{\left(W_{j}, \Phi_{j}, v_{j}\right)\right\}_{j \in J}$ is a $K$-g-fusion frame with bounds $\left(\frac{(1-a) \sqrt{A}-c}{1+b}\right)^{2}$ and $\left(\frac{(1+a) \sqrt{B}+c\|K\|}{1-b}\right)^{2}$ for $\mathcal{H}$.

Theorem 3.18 Let $K \in L(\mathcal{H})$ have closed range and $\Lambda=\left\{\left(W_{j}, \Lambda_{j}, v_{j}\right)\right\}_{j \in J}$ be a $K$-g-fusion frame with bounds $A$ and $B$. Suppose $\Phi_{j} \in L\left(\mathcal{H}, W_{j}\right), j \in J$. If there exist constants $a, b, c$ satisfying $0 \leq a, b<1,0 \leq \frac{c}{1-a}<\frac{\sqrt{A}}{\left\|K^{\dagger}\right\|}$ and such that for any $f \in \mathcal{H}$,

$$
\begin{aligned}
\left(\sum_{j \in J} v_{j}^{2}\left\|\left(\Lambda_{j} \pi_{W_{j}}-\Phi_{j} \pi_{W_{j}}\right) f\right\|^{2}\right)^{\frac{1}{2}} \leq & a\left(\sum_{j \in J} v_{j}^{2}\left\|\Lambda_{j} \pi_{W_{j}} f\right\|^{2}\right)^{\frac{1}{2}} \\
& +b\left(\sum_{j \in J} v_{j}^{2}\left\|\Phi_{j} \pi_{W_{j}} f\right\|^{2}\right)^{\frac{1}{2}}+c\|f\|,
\end{aligned}
$$


then $\Phi=\left\{\left(W_{j}, \Phi_{j}, v_{j}\right)\right\}_{j \in J}$ is also a $K$-g-fusion frame with bounds $\left(\frac{(1-a) \sqrt{A}-c\left\|K^{\dagger}\right\|}{1+b}\right)^{2}$ and $\left(\frac{(1+a) \sqrt{B}+c}{1-b}\right)^{2}$.

Proof The proof is similar to that of Theorem 3.17.

\section{Acknowledgements}

The authors would like to thank the editor and the referees for their valuable comments and suggestions, which improved the quality of our paper.

\section{Funding}

This work is supported by the National Natural Science Foundation of China (Nos. 11761001 and 11761003 ), Leading Talent Project of Science and Technology Innovation of NingXia (No. KJT2016002), Major Project of North Minzu University (No. ZDZX201801) and the graduate innovation project of North Minzu University (No. YCX19123).

Availability of data and materials

Not applicable.

\section{Competing interests}

The authors declare that they have no competing interests.

\section{Authors' contributions}

The authors contributed equally and significantly in writing this paper. The authors read and approved the final manuscript.

\section{Publisher's Note}

Springer Nature remains neutral with regard to jurisdictional claims in published maps and institutional affiliations.

\section{Received: 11 July 2019 Accepted: 19 February 2020 Published online: 28 February 2020}

\section{References}

1. Arabyani, N.F., Arefijamaal, A.A.: Characterization and construction of $K$-fusion frames and their duals in Hilbert spaces. Results Math. 73(1), 47 (2018)

2. Bhandari, A., Mukherjee, S.: Atomic subspaces for operators (2017) arXiv:1705.06042

3. Casazza, P.G.: The art of frame theory. Taiwan. J. Math. 32(46), 1-72 (1999)

4. Casazza, P.G., Kutyniok, G.: Frames of subspaces. Wavelets Frames Oper. Theory 8(3), 87-113 (2003)

5. Casazza, P.G., Kutyniok, G.: Finite Frames: Theory and Applications. Springer, USA (2013)

6. Casazza, P.G., Kutyniok, G., Li, S.: Fusion frames and distributed processing. Appl. Comput. Harmon. Anal. 25(1), 114-132 (2008)

7. Christensen, O.: An Introduction to Frames and Riesz Bases. Springer, Berlin (2003)

8. Daubechies, I., Heil, C.: Ten lectures on wavelets. J. Comput. Phys. 6(3), 1671 (1998)

9. Douglas, R.G.: On majorization, factorization, and range inclusion of operators on Hilbert space. Proc. Am. Math. Soc. 17(2), 413-415 (1966)

10. Duffin, R.J., Schaeffer, A.C.: A class of nonharmonic Fourier series. Trans. Am. Math. Soc. 72(2), 341-366 (1952)

11. Găvruta, L.: Frames for operators. Appl. Comput. Harmon. Anal. 32(1), 139-144 (2012)

12. Han, D., Larson, D.R.: Frames, bases and group representations. Mem. Am. Math. Soc. (2000). https://doi.org/10.1090/memo/0697

13. Hua, D.L., Huang, Y.D.: K-g-frames and stability of K-g-frames in Hilbert spaces. J. Korean Math. Soc. 53(6), 1331-1345 (2016)

14. Huang, Q., Zhu, L., Geng, W., Yu, J.: Perturbation and expression for inner inverses in Banach spaces and its applications. Linear Algebra Appl. 436(9), 3721-3735 (2012)

15. Huang, Y.D., Shi, S.N.: New constructions of K-g-frames. Results Math. 73(4), 162 (2018)

16. Li, X.B., Yang, S.Z., Zhu, Y.C.: Some results about operator perturbation of fusion frames in Hilbert spaces. J. Math. Anal. Appl. 421(2), 1417-1427 (2015)

17. Ramu, G., Johnson, P.S.: Frame operators of K-frames. SeMA J. 73(2), 171-181 (2016)

18. Sadri, V., Ahmadi, R., Rahimi, A.: Constructions of K-g-fusion frames and their duals in Hilbert Spaces (2018) arXiv:1806.03595

19. Shi, S.N., Huang, Y.D.: K-g-frames and their dual. Int. J. Wavelets Multiresolut. Inf. Process. 17(3), 195-215 (2019)

20. Sun, W.C.: g-frames and g-Riesz bases. J. Math. Anal. Appl. 322(1), 437-452 (2006)

21. Zhou, Y., Zhu, Y.C.: K-g-frames and dual g-frames for closed subspaces. Acta Math. Sinica (Chin. Ser.) 56(5), 799-806 (2013) 